\title{
Validação transcultural do Teste de Orientação da Vida (TOV-R)
}

\author{
Marina Bandeira \\ Universidade Federal de São João Del-Rey \\ Valentin Bekou \\ Douglas Hospital \\ Keli Silva Lott \\ Marcela Augusta Teixeira \\ Sandra Silva Rocha \\ Universidade Federal de São João Del-Rey
}

\begin{abstract}
Resumo
Esta pesquisa descreve a validação transcultural e as qualidades psicométricas da versão revisada do Teste de Orientação da Vida (TOV-R). Esta escala avalia o construto de otimismo, em termos de expectativas em relação a eventos futuros. O TOV-R foi submetido a uma "backtranslation", estudo piloto e análise das propriedades psicométricas. Participaram desta pesquisa 396 estudantes universitários, que responderam ao TPV e ao Inventário de Depressão de Beck. Após 30 dias, uma sub-amostra aleatória de 48 sujeitos foi submetida a um reteste da escala. Os resultados mostraram uma correlação positiva significativa teste-reteste $(\mathrm{r}=0,61)$ e uma consistência interna adequada $(\alpha=0,68)$. O grau de otimismo foi preditivo de menor presença de sintomas depressivos $(\mathrm{r}=-0,42)$. Uma análise fatorial exploratória indicou a presença de um único fator composto por seis itens, que explicaram $39,78 \%$ da variância. Os resultados indicaram boas qualidades psicométricas de fidedignidade e validade para a versão brasileira do TOV-R.
\end{abstract}

Palavras-chave: Teste de Orientação da Vida, Propriedades psicométricas, Validação transcultural.

\begin{abstract}
Transcultural validation of the Life Orientation Test (LOT-R). The transcultural validation of the Revised Life Orientation Test (LOT-R) was implemented and the psychometric properties of this test were examined in the Brazilian context. This test evaluates the construct of dispositional optimism, in terms of outcome expectancies concerning future life events. The LOT-R was first submitted to a back translation and to a pilot study in order to make its transcultural adaptation. Then, a sample of 396 university students answered this test and the Beck Depression Inventory (BDI). A retest was implemented 30 days later in a randomly selected sub-sample of 48 students. Results showed a significant test-retest correlation $(\mathrm{r}=0,61)$ and good internal consistency $(\alpha=0,68)$. Degree of optimism was predictive of fewer degree of depressive symptoms $(r=-0,42)$. Exploratory factorial analysis identified one factor explaining 39,78\% of variance. The results demonstrated good psychometric properties for the Brazilian version of the LOT.
\end{abstract}

Key words: Life Orientation Test (LOT-R), Transcultural Validation, Psychometric Properties.

$\mathrm{O}$ Teste de Orientação da Vida (Life Orientation TestLOT, Scheier \& Carver, 1985; Scheier \& Carver, 1992) visa medir o construto de orientação da vida, referente à maneira como as pessoas percebem suas vidas, de uma forma mais otimista ou menos otimista. Este construto foi definido em termos das expectativas que as pessoas possuem sobre os eventos que ocorrerão no futuro em suas vidas. Este conceito está inserido na teoria de auto-regulação do comportamento, desenvolvida pelos memos autores, se- gundo a qual as pessoas lutam para alcançar objetivos quando elas acreditam que estes objetivos sejam possíveis e que suas ações produzirão os efeitos desejados nesta direção (Hjelle, Belongia \& Nesser, 1996).

O TOV se correlaciona com medidas de outros conceitos correspondentes, tais como auto-estima, locus de controle, auto-eficácia, estilo atribucional e neuroticismo, mas o conceito de percepção otimista da vida medido através do TOV se diferencia destes outros conceitos, como um fator distin- 
to, tal como tem sido evidenciado através de pesquisas desenvolvidas com o objetivo de confrontar estes diversos instrumentos (Scheier \& Carver, 1992; Scheier \& Bridges, 1994).

O TOV tem sido utilizado principalmente no contexto clínico. Pesquisas têm relacionado a orientação otimista da vida com o bem estar psicológico e físico das pessoas, com a presença de comportamentos de manutenção da saúde e com a capacidade das pessoas de enfrentamento (coping) em situações estressantes (Scheier \& Carver, 1992; Scheier \& Carver, 1987). Uma orientação otimista está relacionada com saúde física e mental, enquanto que uma orientação pessimista da vida se relaciona com depressão, ansiedade e prática de comportamentos de risco. Estudos prospectivos têm mostrado, por exemplo, que o pessimismo constitui um fator preditivo de depressão pós-parto. Do mesmo modo, observou-se uma relação entre o construto de orientação otimista da vida e a adaptação de pacientes cardíacos em situações de recuperação pós-operatória, em uma pesquisa prospectiva. Pacientes cardíacos que haviam apresentado anteriormente maior grau de otimismo, medido pelo TOV, obtiveram uma melhora pós-operatória mais rápida, em termos de indicadores fisiológicos e comportamentais (Scheier \& Carver, 1992). Foi observado igualmente que o conceito de otimismo, medido pelo TOV, foi preditivo do nível de adaptação ao diagnóstico e tratamento de câncer de mama (Carver et al., 1994).

O conceito de orientação otimista da vida tem sido igualmente estudado no contexto educacional, tendo sido relacionado com a capacidade de adaptação e desempenho escolar. Um nível baixo de otimismo foi preditivo de dificuldades de adaptação ao ambiente acadêmico, em estudantes universitários, em termos de sintomas depressivos, sentimentos de solidão e sintomas de estresse, ao longo do primeiro ano do curso, assim como menor desempenho acadêmico nos anos posteriores (Scheier \& Carver, 1987; Vickers \& Vogeltanz, 2000). Scheier et al. (1994), em um estudo transversal de uma amostra de estudantes universitários, encontraram que um maior grau de otimismo era preditivo de menor grau de sintomas depressivos, medido através do Inventário de Beck. Vickers e Vogeltanz (2000) confirmaram estes dados, em um estudo longitudinal, tendo observado que o grau de otimismo apresentado por estudantes universitários foi preditivo de menor grau de sintomas depressivos apresentados após 10 meses.

Há algumas evidências de que o estilo de enfrentamento intermedia a relação entre o grau de otimismo e o bem-estar físico e psicológico. Estilos diferentes de enfrentamento têm sido relacionados a pessoas com diferentes níveis de otimismo. Pessoas mais otimistas apresentam estratégias adaptativas de enfrentamento de situações adversas, em um estilo ativo de enfrentamento (active copers). Por outro lado, pessoas pessimistas apresentam um estilo de enfrentamento caracterizado pela evitação (avoidant copers, incluindo uma negação da situação real e um desengajamento da situação, em termos de uma ausência de ações concretas para resolver a situação (Scheier \& Carver, 1992).

Alguns estudos relacionaram o conceito de otimismo com o conceito de estilo de atribuição, este último derivado de um modelo cognitivo (Learned helplessness, Seligman,
Abramson, Semmel \& von Baeyer, 1979), segundo o qual as explicações causais que as pessoas adotam a respeito de eventos passados em suas vidas influenciam suas expectativas em relação a eventos futuros. Atribuições de que eventos negativos possuem causas internas, estáveis e globais estariam relacionadas a uma orientação pessimista. Ao contrário, atribuições de eventos negativos a causas externas, instáveis e específicas estariam relacionadas a uma orientação mais otimista. A diferença entre estes dois conceitos é que o TOV avalia as expectativas das pessoas em relação a eventos futuros e a medida de estilo de atribuição avalia as explicações causais das pessoas em relação aos eventos. As avaliações destes dois conceitos têm apresentado correlações moderadas, indicando que se trata de conceitos correlatos porém distintos (Scheier \& Carver, 1992).

O teste TOV foi submetido a uma revisão recentemente, na qual foram eliminados itens que não se relacionavam a processos de expectativas, tornando-se um teste mais curto e mais homogêneo, que apresentou propriedades psicométricas adequadas de validade preditiva e consistência interna satisfatória, com $\alpha=0,78$ (Scheier et al., 1994; Vichers \& Vogestanz, 2000), fidedignidade temporal teste-resteste adequada $(\mathrm{r}=0,76)$ e validade concomitante e discriminante (Hielle et al., 1996; Scheier et al.; 1994; Lai, Cheung, Lee \& Yu (1998). Lai et al. (1998) consideraram que a versão revisada desta escala (TOV-R) apresentava propriedades psicométricas mais adequadas do que a versão original.

No que se refere à estrutura fatorial do TOV, tem havido uma controvérsia em torno da presença de um fator ou dois fatores, uma vez que diferentes resultados têm sido obtidos nas pesquisas. Alguns autores consideraram o TOV como uma escala unidimensional bipolar, tendo otimismo e pessimismo como dois polos opostos de uma mesma dimensão ou construto, enquanto outros consideraram que havia dois fatores distintos. No estudo original (Scheier \& Carver, 1985), a análise fatorial exploratória identificou a presença de 2 fatores correlacionados $(r=-0,64)$, mas a análise fatorial confirmatória indicou que os dois modelos possíveis de estrutura fatorial (com 1 ou 2 fatores) eram igualmente adequados, os autores tendo então considerado mais parcimonioso tratar o TOV como um teste unidimensional. O estudo do teste revisado na língua inglesa (LOT-R) resultou em uma estrutura dimensional de um único fator, que explicou 48,1\% da variância dos dados (Scheier et al., 1994). Lai et al. (1998) também encontraram uma estrutura unidimensional no TOV revisado, ao fazer uma análise fatorial confirmatória. Hjelle et al. (1996) sugeriram igualmente que esta escala poderia ser considerada como unidimensional, pois em seu estudo os itens positivos e os itens negativos obtiveram uma correlação negativa de $-0,53$.

Por outro lado, Myers e Steed (1999) consideraram que o TOV, na sua forma original, consistia em uma escala de dois fatores, uma vez que estes fatores, em seu estudo, correlacionaram diferentemente com medidas de um outro construto referente ao estilo de enfrentamento (Repressive coping). Além disso, estes autores consideraram baixa a correlação de $r=-0,50$ obtida por eles entre os dois fatores, assim 
como as correlações obtidas em outras pesquisas, as quais têm alcançado valores na faixa de $-0,47$ a $-0,57$, entre os dois fatores, sugerindo que os itens de pessimismo e de otimismo do TOPV seriam construtos diferentes e não extremos opostos de um mesmo construto. Entretanto, estes resultados, que indicaram a presença de uma estrutura com dois fatores, podem ter sido determinados pela forma de redação dos itens do TOV, metade deles redigido em termos negativos e metade em termos positivos. Chang e McBride-Chang (1996) demostraram que, de fato, quando se alterou a formulação das questões de forma a uniformizá-las, os resultados indicaram uma estrutura unidimensional.

Os resultados obtidos nas diversas pesquisas mostram que a versão revisada do TOV tem produzido resultados diferentes dos obtidos com sua versão original, no que se refere à estrutura dimensional, resultando em análises que reforçam mais a estrutura unidimensional do teste. Torna-se necessário, portanto, mais estudos sobre a estrutura dimensional da versão revisada do TOV em diferentes meios e culturas, afim de esclarecer este aspecto.

O TOV-R foi traduzido, adaptado e validado para a cultura chinesa, tendo sido utilizado em uma amostra de 248 estudantes chineses. Neste caso, a versão revisada apresentou qualidades psicométricas adequadas de validade concomitante e discriminante, assim como de fidedignidade, apresentando, além disso, na análise fatorial confirmatória, uma estrutura dimensional claramente unidimensional (Lai et al., 1998). O TOV foi igualmente traduzido, adaptado e validado para a cultura canadense-francesa, tendo apresentado igualmente propriedades psicométricas adequadas de validade e fidedignidade, mas uma estrutura bifatorial (Bekou, Vallerand \& Tetreau, 1998; Bekou, Tetreault, Valerand \& Gouzet, 1999). No Brasil, do nosso conhecimento, este teste ainda não foi validado.

$\mathrm{O}$ presente trabalho visa preencher esta lacuna, apresentando a adaptação brasileira do TOV-R e a análise de suas propriedades psicométricas, com o objetivo de estender $\mathrm{O}$ estudo e a aplicação deste teste para uma outra cultura.

\section{Método}

\section{Participantes}

Para estudar as propriedades psicométricas da versão brasileira do TOV-R, foi utilizada uma amostra de sujeitos que participou de uma primeira aplicação do teste e uma subamostra que participou também de uma replicação do teste. Estas amostras serão descritas a seguir.

Amostra da Primeira aplicação: A amostra dos sujeitos que participaram da primeira aplicação do TOV-R foi composta por estudantes universitários da Fundação de Ensino Superior de São João del-Rei (FUNREI), de ambos os sexos, que cursavam os cursos de: Administração, Ciências, Engenharia Elétrica, Engenharia Mecânica, Filosofia, Economia, Pedagogia, Psicologia e Letras, estando matriculados em diversos períodos dos cursos.

Participaram dessa primeira aplicação 396 alunos, com idade média de 22,34 anos, sendo 188 do sexo masculino $(47,5 \%)$ e 207 do sexo feminino $(52,3 \%)$,
Sub-amostra do reteste: Para a realização do reteste, foi composta uma sub-amostra de 48 sujeitos, com idade média de 23,27 anos, selecionados aleatoriamente dentre os estudantes que participaram da primeira amostra. Esta sub-amostra consistia de 24 homens (50\%) e 24 mulheres (50\%).

\section{Instrumentos de medida}

Para verificar as propriedades psicométricas da versão brasileira do Teste de Orientação da Vida, os sujeitos responderam a dois instrumentos de medida, o TOV-R e o Inventário de Depressão de Beck, visando investigar se um maior grau de otimismo (predictor), medido pelo TOV-R, seria preditivo de um menor grau de sintomas depressivos desenvolvidos pelos sujeitos (outcome), medido através do Inventário do Beck (Scheier et al., 1994). Apresentamos a seguir uma descrição dos dois testes.

Teste de Percepção da Vida: Foi utilizada a versão revisada canadense-francesa do Teste de Orientação da Vida, traduzido e adaptado para a cultura canadente-francesa por Bekou et al. (1998) e validado por Bekou et al. (1999). Esta versão apresentou qualidades psicométricas satisfatórias, em termos de uma boa consistência interna (alpha de Cronbach $=0,68)$, estabilidade temporal teste-reteste $(r=0,61)$ e uma estrutura dimensional de dois fatores explicando $56,58 \%$ da variância dos dados. Os coeficientes de saturação variaram de 0,48 a 0,82. O teste TOV-R apresentou igualmente qualidades psicométricas satisfatórias nas suas versões chinesa e de língua inglesa, conforme descrito acima, na introdução.

A versão utilizada no presente trabalho corresponde à versão revisada, contendo dez itens (Scheier et al., 1994). Dentre esses itens, encontram-se três afirmativas positivas (itens 1, 4 e 10), três afirmativas negativas (itens 3, 7 e 9) e quatro questões neutras $(2,5,6$ e 8$)$. As questões neutras (filler questions) não visam analisar o construto de orientação da vida e, portanto, não são incluídos na análise de dados.

Ao responder o questionário, o sujeito deve avaliar cada afirmativa em uma escala tipo Likert de 5 pontos, com gradações de 0 a 4, conforme o seu grau de concordância ou discordância em relação à mesma (ver Apêndice). Nesta escala, os valores possuem a seguinte correspondência: $0=$ discordo totalmente, $1=$ discordo, 2 = neutro, $3=$ concordo e 4=concordo totalmente. Para a análise estatística dos dados, os escores dos itens negativos do teste precisam ser invertidos, de modo que todos os valores próximos a 4 indiquem sempre um maior grau de expectativa otimista do sujeito em relação à vida. Após a inversão dos escores dos itens negativos, pode-se calcular o índice global de grau de otimismo através da soma dos seis itens do TPV.

Inventário de Depressão de Beck: O Inventário de Depressão de Beck contém 21 questões que visam avaliar a presença de sintomas depressivos. Cada questão é formada por quatro alternativas, as quais descrevem traços que caracterizam o quadro depressivo. Ao responder ao teste, o sujeito opta por uma destas alternativas, que variam de 0 a 3 . A afirmativa que equivale a 0 na escala corresponde à ausência de sintomas depressivos e as outras descrevem gradativamente 
traços que indicam a presença cada vez maior de sintomas depressivos. Este teste avalia o grau de sintomas depressivos dos sujeitos em relação ao período da semana anterior à aplicação do teste (Gorestein \& Andrade, 1998).

Vários estudos confirmaram as qualidades psicométricas deste teste, em termos de sua fidedignidade teste-reteste, homogeneidade, sensibilidade a mudanças e a estrutura fatorial com 4 fatores principais, em sua versão inglesa (Beck \& Beamesderfer, 1974; Beck, Steer \& Garbin, 1988). Estudos franceses confirmaram sua estrutura fatorial e demonstraram sua validade concomitante (Collet \& Cottraux, 1986; Lemperière et al., 1984).

A versão brasileira do Inventário de Beck foi validada por Cunha, Prieb, Goulart e Lemes (1996), tendo sido demonstrada as suas propriedades psicométricas de fidedignidade teste-reteste $(r=0,40 ; p<0,001)$ e consistência interna $(\alpha=$ $0,84)$, assim como sua validade discriminante , pela sua capacidade de diferenciar pacientes deprimidos de ansiosos e de sujeitos normais (Gorestein \& Andrade, 1998). Sua estrutura fatorial conta com 3 fatores principais responsáveis respectivamente por $28,3 \%, 6,4 \%$ e $6,1 \%$ da variância dos dados (Gorestein \& Andrade, 1998).

\section{Procedimento}

Adaptação transcultural do Teste de Percepção da Vida: A validação transcultural do TOV-R para o contexto brasileiro foi realizada segundo as recomendações de Vallerand (1989). Assim, a versão brasileira inicial do TOV-R foi elaborada a partir de uma tradução reversa (backtranslation) realizada por duas pessoas bilíngües. Em primeiro lugar, uma psicóloga bilíngüe de nacionalidade brasileira traduziu o TOV-R do original em francês para o português. Em seguida, uma segunda pessoa bilíngüe de nacionalidade francesa, fez a retrotradução, vertendo para o francês. As duas formas em francês, a original e a retrotraduzida, foram então comparadas por um grupo composto pelos dois tradutores e por uma outra psicóloga brasileira. Estas três pessoas discutiram a respeito das inconsistências encontradas e chegaram a um consenso para a correção e redação das questões em português.

Esta versão inicial do consenso foi então aplicada em uma amostra de 20 estudantes universitários para se verificar a compreensão dos termos utilizados. A cada aplicação em pequenos grupos de 4 a 6 sujeitos, seguia-se uma discussão com os sujeitos a respeito de possíveis incompreensões dos itens do TPV, em função das formulações das frases. As críticas e sugestões de cada sujeito eram então anotadas e discutidas pelo grupo de especialistas, resultando em modificações do teste. As reformulações foram incorporadas e o teste foi reaplicado a outro pequeno grupo de sujeitos, até que não se encontrou mais problemas de compreensão na sua aplicação, tornando-se portanto mais claro e fácil de ser compreendido pela população-alvo. A versão final do TOV-R para o contexto brasileiro (ver Apêndice) foi então aplicada nas amostras de estudantes universitários, descritas acima, com o objetivo de analisar suas propriedades psicométricas.

Coleta de dados: Com a finalidade de se validar o Teste de Orientação da Vida para o contexto brasileiro, foram aplicados os dois instrumentos de medida descritos acima, na amostra de estudantes universitários da FUNREI.

A aplicação dos testes foi realizada de forma coletiva em salas de aula, por dois assistentes de pesquisa. A aplicação dos testes seguiu um procedimento padronizado, descrito a seguir. Primeiramente, os alunos eram informados sobre a finalidade da pesquisa de validação transcultural e sobre os objetivos dos testes. Explicava-se que o teste TOV-R avaliava a percepção da pessoas sobre a vida ou a maneira como as pessoas se orientam na vida, através de questões de múltipla escolha e que o Inventário de Beck avaliava os sentimentos das pessoas, também através de questões de múltipla escolha. Explicava-se, em seguida, a maneira como os sujeitos deviam responder a cada questão, escolhendo a resposta que mais correspondesse à sua percepção e marcando-a com um X. Solicitava-se que respondessem de forma honesta, segundo sua percepção e seus sentimentos e que não deixassem sua resposta a uma questão influenciar suas respostas às outras questões (Scheier et al., 1994). Explicava-se ainda que não havia respostas certas ou erradas e garantia-se a confidencialidade dos resultados.

Após um intervalo de trinta dias, foi realizado o reteste em uma sub-amostra dos sujeitos, que participaram então de uma segunda aplicação do TOV-R. O reteste foi realizado com o objetivo de verificar a fidedignidade ou estabilidade temporal do instrumento. Foram repetidos os procedimentos de aplicação descritos acima. Os resultados foram submetidos a uma análise correlacional para se verificar a estabilidade temporal da escala.

A análise das propriedades psicométricas do TOV-R incluiu também uma análise fatorial exploratória, com rotação Varimax, para se verificar a estrutura fatorial do teste. Além disso, foi feita uma análise correlacional entre o Inventário de Beck e o TOV-R, com o objetivo de avaliar se o grau de otimismo seria preditivo de menor grau de sintomas depressivos apresentados pelos sujeitos. Foi postulada, portanto, uma correlação negativa entre estes dois testes. A consistência interna do TOV-R foi igualmente avaliada através da análise do coeficiente alpha de Cronbach. Foi utilizado o programa estatístico SPSS-PC para a realização das análises estatísticas.

\section{Resultados}

Os resultados referentes à fidedignidade e à validade da versão brasileira do TOV-R, obtidos a partir das análises estatísticas citadas acima, serão descritos a seguir.

\section{Estabilidade temporal}

A fidedignidade do TOV-R foi verificada através de uma análise correlacional de Pearson entre os escores das duas aplicações teste-reteste, realizadas com um intervalo de 30 dias, na sub-amostra de 48 sujeitos. A Tabela 1 apresenta as médias e os desvios-padrão obtidos nestas duas aplicações, referentes aos seis itens positivos e negativos, assim como o valor do coeficiente de correlação obtido.

Pode-se observar, na Tabela 1, que há uma correlação positiva significativa $(r=0,61, p<0,001)$ entre os dados do teste e do reteste. Esse resultado demonstra que o Teste de 
Tabela 1.

Médias e desvios-padrão dos escores do TOV-R no teste e no reteste e coeficiente de correlação de Pearson entre os dois escores.

$\begin{array}{llll}\text { Aplicações do TOV-R N } \quad \text { Médias } \quad \text { Desvios-Padrão } & \begin{array}{c}\text { Correlação } \\ \text { Teste-reteste }\end{array}\end{array}$

\begin{tabular}{ccccc}
\hline TOV-R teste & 48 & 17,87 & 3,18 & \\
TOV-R reteste & 48 & 17,89 & 4,02 & $0,61^{* *}$ \\
\hline
\end{tabular}

$* * \mathrm{p}<0,001$

Orientação da Vida é um instrumento de medida que apresenta estabilidade temporal ou fidedignidade para medir o construto de orientação da vida.

\section{Consistência Interna}

A consistência interna do TOV-R foi verificada através do cálculo do coeficiente alpha de Cronbach aplicado aos seis itens positivos e negativos do teste. A Tabela 2 mostra os dados referentes a esta análise. Podemos observar uma consistência interna satisfatória, uma vez que o valor de alpha foi elevado $(\alpha=0,68)$.

As correlações item-total variaram de 0,31 a 0,52 , indicando que cada item está medindo parcialmente o mesmo construto subjacente, sem ser redundante com os demais itens. Além disso, podemos observar, na segunda coluna da Tabela 2, que os itens contribuíram igualmente para o valor de alfa de Cronbach, pois as diminuições dos valores de alfa foram relativamente comparáveis, à medida que os itens eram individualmente removidos da escala.

Estes resultados indicam que os itens do teste TOV-R apresentaram uma boa homogeneidade ou fidedignidade.

\section{Relação entre otimismo e depressão}

A relação inversa entre o grau de otimismo, medido através do TOV, e o grau de depressão, teoricamente prevista, foi avaliada através de uma análise correlacional de Pearson en- tre os escores do TOV-R e os escores do Inventário de Beck. A Tabela 3 apresenta as médias e os desvios-padrões dos escores obtidos através do TOV-R e do Inventário de Beck, assim como o coeficiente de correlação encontrado entre eles.

Os resultados desta análise mostraram uma correlação negativa significativa entre os escores dos dois testes $(r=-$ 0,42, $\mathrm{p}<0,001$ ). O grau de otimismo, medido pelo TOV-R foi, portanto, uma variável preditiva da presença de um menor nível de sintomas depressivos na amostra estudada. Ou seja, quanto maior era o grau de otimismo ou percepção positiva que os sujeitos possuíam a respeito da vida, menor era o número de sintomas depressivos apresentados por eles.

\section{Validade de Construto}

Os dados dos itens positivos e negativos do TOV-R foram submetidos a uma análise fatorial exploratória, com rotação Varimax. Os resultados desta análise indicaram a presença de um único fator, composto por seis itens, que avaliam o construto de Percepção da Vida. A porcentagem de variância explicada por este fator foi de $39,78 \%$. O fator identificado apresentou um valor do Eingenvelue de 2,38. Os demais fatores mostraram-se irrelevantes, pois os seus respectivos Eingenvalues se situavam abaixo de 1,0.

A Tabela 4 apresenta os valores dos coeficientes de saturação dos seis itens que compõem o fator. Pode-se observar que os coeficientes de saturação destes itens variaram

Tabela 2.

Consistência interna da versão brasileira do TOV-R, através do coeficiente alpha de Cronbach.

\begin{tabular}{ccc|}
\hline Itens do TOV-R & $\begin{array}{c}\text { Correlações } \\
\text { item-total }\end{array}$ & $\begin{array}{c}\text { Valores de alpha } \\
\text { se os itens são removidos }\end{array}$ \\
\hline Item 1 & 0,34 & 0,66 \\
Item 3 & 0,31 & 0,67 \\
Item 4 & 0,48 & 0,61 \\
Item 7 & 0,33 & 0,66 \\
Item 9 & 0,52 & 0,60 \\
Item 10 & 0,51 & 0,66 \\
\hline Alpha $=0,68$ & &
\end{tabular}


Tabela 3.

Médias e desvios-padrão dos escores do TOV-R e do Inventário de Depressão de Beck e coeficiente de correlação de Pearson entre os escores dos dois testes.

\begin{tabular}{ccccc}
\hline Testes & $\mathrm{N}$ & Médias & Desvios-padrão & Correlação \\
\hline TOV-R & 396 & 17,66 & 3,76 & \\
BECK & 396 & 9,86 & 6,98 & $-0,42^{* *}$ \\
\hline
\end{tabular}

$* * \mathrm{p}=0,001$

entre 0,49 e 0,73. Foi adotado o critério de 0,30 como valor numérico mínimo para o coeficiente de saturação, abaixo do qual o item seria eliminado. Os resultados da análise fatorial comprovaram a adequação da amostra, uma vez que o valor de KMO foi igual a 0,79. O teste de Bartlett apresentou resultados significativos (Qui-quadrado $=325,44 ; \mathrm{df}=15, \mathrm{p}<0,001$ ), indicando a adequação da matrix de correlação.

\section{Discussão}

Os resultados obtidos no presente trabalho mostraram que a versão brasileira do Teste de Orientação da Vida (TOV$\mathrm{R})$ apresentou qualidades psicométricas satisfatórias, em termos de sua fidedignidade e validade.

A consistência interna da versão brasileira $(\alpha=0,68)$ atingiu um resultado idêntico $(\alpha=0,68)$ ao obtido na versão canadense-francesa (Bekou et al., 1999), embora este valor esteja abaixo do resultado obtido $(\alpha=0,78)$ para a versão revisada de língua inglesa (Scheier et al., 1994). O resultado obtido na amostra brasileira se aproxima igualmente dos dados obtidos $(\alpha=0,70)$ na amostra chinesa (Lai et al., 1998).

A estabilidade temporal da versão brasileira foi satisfatória, tendo atingido uma correlação significativa teste-reteste de 0,61 , idêntica ao resultado obtido para a versão canadense-francesa $(r=0,61)$ e dentro da faixa encontrada por Scheier et al. (1994) para quatro amostras de estudantes $(0,56$ a 0,79$)$, embora este valor tenha sido inferior ao obtido ( $r$ $=0,76$ ) com a versão revisada de língua inglesa, no estudo de Hjelle et al. (1996). O resultado obtido na amostra brasileira se aproxima igualmente dos dados obtidos $(\mathrm{r}=0,66)$ na amostra chinesa (Lai et al., 1998).

Além disso, foi obtida uma correlação negativa significativa entre o TOV-R e o teste do Beck $(r=-0,42, p<0,001)$, demonstrando assim, tal como previamente postulado, que o grau de otimismo é uma variável preditiva de menor presença de sintomas depressivos. Ou seja, quanto maior era o grau de otimismo ou expectativa positiva a respeito dos eventos futuros da vida, menor era o nível de sintomas depressivos apresentados pelos sujeitos. Estes resultados são idênticos aos dados obtidos para a versão revisada de língua inglesa, que também apresentaram uma correlação negativa significativa entre estes dois testes $(r=-0,42 ; p<0,001)$, em um estudo transversal com estudantes universitários (Scheier et al., 1994). Estes resultados foram confirmados em um estudo longitudinal (Vickers \& Vogeltanz, 2000), em um período de 10 meses, no qual se constatou que o grau de otimismo foi preditivo dos níveis futuros de sintomas depressivos, em uma amostra de estudantes universitários.

Quanto à estrutura dimensional do TOV-R, os resultados da versão brasileira identificaram a presença apenas de um fator, explicando 39,78\% da variância dos dados, diferentemente da versão canadense-francesa, que apresentou uma estrutura dimensional com dois fatores, explicando 56,58\% da variância. Neste caso, os resultados obtidos aqui para a versão brasileira do TOV-R estão mais em acordo com os dados encontrados para a versão revisada de língua inglesa, que também apresentaram uma estrutura fatorial de apenas

Tabela 4 .

Coeficientes de saturação dos itens positivos e negativos do TOV-R.

\begin{tabular}{lc}
\hline Itens & Coeficientes de saturação \\
\hline Item 1 & 0,55 \\
Item 3 & 0,49 \\
Item 4 & 0,71 \\
Item 7 & 0,53 \\
Item 9 & 0,73 \\
Item 10 & 0,73 \\
\hline
\end{tabular}

Eigenvalue $=2,38$

$\%$ Variancia total $=39,78$ 
um fator (Scheier et al., 1994). Do mesmo modo, a versão chinesa do TOV-R também apresentou uma estrutura unidimensional, após uma análise fatorial confirmatória (Lai et al., 1998). Hjelle et al. (1996) argumentaram também a favor da estrutura unidimensional do TOV-R, com base na correlação significativa encontrada entre os itens negativos e os itens positivos da escala.

Segundo Lai et al., (1998) estruturas similares encontradas para o TOV-R em diferentes culturas fornecem condições significativas para a realização de pesquisas transculturais. O presente trabalho, tendo realizado a adaptação transcultural desta escala para o contexto brasileiro, segundo os critérios indicados por Vallerand (1989) e, tendo constatado qualidades psicométricas satisfatórias para a versão brasileira do TOV-R, contribuirá portanto para a realização de pesquisas transculturais em nosso meio, envolvendo, entre outras, a avaliação do grau de otimismo e suas relações com variáveis clínicas.

\section{Referências}

Beck, A. T., \& Beamesderfer, A. (1974). Assessment of depression: the Depression Inventory. Psychological Measurements in Psychopharmacology: Modern Problems in Pharmacopsychiatry, 7, 151-159.

Beck, A. T., Steer, R., \& Garbin, M. (1988). Psychometric properties of the Beck Depression Inventory: twenty-five years of research. Clinical Psychology Review, 8, 77-100.

Bekou, V., Vallerand, R., \& Tetreau B. (1998). Le test d'orientation de la vie: version canadienne française du Life Orientation Test. Université de Montréal. Documento não-publicado.

Bekou,V., Tetreault,B., Vallerand, R., \& Gouzet, F. (1999). Developpement et validation d' une version canadienne-francaise du "Life Orientation Test". Trabalho apresentado no Congrès de la Societé Québecoise de Recherche en Psychologie, Montréal, Canada.

Carver, C. S., Pzokaderman, C., Harris, S. D., Noriega, V., Scheier, M. F., Robinson, D. S., Ketcham, A. S., Moffat, F. L., \& Clark, K. C. (1994). Optimism versus pessimism predicts the quality of womens adjustement to early-stage breast-cancer. Cancer, 73(4), 1213-1220.

Chang, L., \& Mcbride-Chang, C. (1996). The factor structure of the life orientation test. Educacional and Psychologial Measurement. 56(2), 325-329.

Collet, L., \& Cottraux, J. (1986).Inventaire abrégé de la dépression de Beck: étude de la validité concurrente avec les échelles de Hamilton et de ralentissement de Widlöcher. L'Encéphale, 12, 77-79.
Cottraux, J., Bouvard, M., \& Legeron, P. (1985). Méthodes et échelles d'évaluation des comportements. Issy-les-Moulineaux: Editions d'Applications Psychotechniques.

Cunha, J. A., Barraz, A. C. G., Lemes, R. B., Brenner, M. K., Prieb, R. G. G., \& Goulart, P. M. (1995). Notas preliminares de um estudo sobre depressão e ansiedade em estudantes universitários. Psico, 26(1), 143-150.

Cunha, J. A., Prieb, R. G. G., Goulart, P. M., \& Lemes, R.B. (1996). O uso do Inventário de Beck para avaliar depressão em universitários. Psico, 27(1), 107-115.

Cunha, J. A., Prieb, R. G. G., Touginha, L. A., \& Goulart, P. M. (1996). Depressão em universitários da PUCRS. Psico, 27(2), 97-109.

Dela Coleta, J. A. (1980). Atribuições de causalidade em presos, cegos e amputados. Tese de Doutorado não-publicada. Fundação Getúlio Vargas, Rio de Janeiro.

Gorestein, C., \& Andrade, L. (1998). Inventário de Depressão do Beck: propriedades psicométricas da versão em português. Revista de Psiquiatria Clínica, 25, 245-250.

Hjelle, L., Belongia, C., \& Nesser, J (1996). Psychometric properties of the life orientation test and attributional style questionnaire. Psychological Reports, $78,507-515$.

Lai, J. C. L., Cheung, H., Lee,W. E., \& Yu, H. (1998) The utility of the revised life orientation test to measure optimism among Hong Kong chinese. International Journal of Psychology, 33(1), 45-56.

Lemperiere, T., Lepine, J. P., Rouillon, F., Hardy, P., Ades, J., Luaute, J. P., \& Ferrandi I. (1994). Comparaison de différents instruments d'évaluation de la dépression à l'occasion d'une étude sur l'Athymil $30 \mathrm{mg}$. Ann. MédicoPsychol., 142, 1206-1212.

Scheier, M. F., \& Carver, C. S. (1985). Optimism, coping, and health: assessment and implications of generalized outcome expectancies. Health Psychology, 4, 219-247.

Scheier, M. F, \& Carver, C. S. (1992). Effects of optimism on psychological and physical well-being: theoretical overview and empirical update. Cognitive Therapy and Research, 16(2), 201-228.

Scheier, M. F., Carver, C. S., \& Bridges, M. W. (1994). Distinguishing optimism from neuroticism (and trait anxiety, self-mastery, and self-esteem) - a reevaluation of the Life Orientation Test. Journal of Personality and Social Psychology, 67(6), 1063-1078.

Seligman, M. E. P., Abramson, L. Y., Semmel, A., \& von Bayer, C. (1979). Depressive atributional styles. Journal of Abnormal Psychology, 88, 242-247.

Vallerand, R. J. (1989). Vers une méthodologie de validation transculturelle de questionnaires psychologiques: implications pour la recherche en langue française. Psychologie Canadienne, 30, 662-680.

Vickers K. S., \& Vogeltanz, N. D. (2000). Dispositional optimism as a predictor of depressive symptoms over time. Personality and Individual Differences, $28,259-272$

Marina Bandeira, doutora pela Université de Montreal, Canadá, é professora do Departamento de Psicologia da Universidade Federal de São João Del-Rey (FUNREI), MG.

Valentin Bekou, doutor pela Université de Montreal, Canadá, é pesquisador do Douglas Hospital, Quebec, Canadá.

Sandra Silva, mestranda pela Universidade Estadual do Rio de Janeiro, é professora do Departamento de Psicologia da FUNREI, MG. Keli Silva Lott e Marcela Augusta Teixeira são psicólogas formadas no curso de Psicologia da Universidade Federal de São João Del-Rey, MG.

Endereço para correspondência: Marina Bandeira, Departamento de Psicologia, Universidade Federal de São João Del-Rey. Praça Dom Helvecio, 74, 36.300-000, São João Del Rei, MG. E-mail: bandeira@funrei.br. 
Apêndice

TESTE DE ORIENTAÇÃO DA VIDA (TOV-R)

\section{INSTRUÇÕES}

Marque com um $\mathrm{X}$ as questões abaixo a respeito de você, indicando o seu grau de acordo, segundo a seguinte escala:

$\begin{array}{ccccc}\begin{array}{c}\text { Discordo } \\ \text { totalmente }\end{array} & \text { Discordo } & \text { Neutro } & \text { Concordo } & \begin{array}{c}\text { Concordo } \\ \text { totalmente }\end{array} \\ \mathbf{0} & 1 & 2 & 3 & 4\end{array}$

Seja o(a) mais sincero(a) possível e procure não deixar sua resposta a uma questão influenciar suas respostas às outras questões. Não há respostas certas nem erradas.

1. Nos momentos de incerteza, geralmente eu espero que aconteça o melhor.

$\begin{array}{lllll}0 & 1 & 2 & 3 & 4\end{array}$

2.É fácil para mim relaxar.

$\begin{array}{lllll}0 & 1 & 2 & 3 & 4\end{array}$

3. Se alguma coisa ruim pode acontecer comigo, vai acontecer.

$\begin{array}{lllll}0 & 1 & 2 & 3 & 4\end{array}$

4. Eu sou sempre otimista com relação ao meu futuro.

$\begin{array}{lllll}0 & 1 & 2 & 3 & 4\end{array}$

5. Eu gosto muito da companhia de meus amigos e amigas.

$\begin{array}{lllll}0 & 1 & 2 & 3 & 4\end{array}$

6. É importante para mim manter-me em atividade.

$\begin{array}{lllll}0 & 1 & 2 & 3 & 4\end{array}$

7. Quase nunca eu espero que as coisas funcionem como eu desejaria.

$\begin{array}{lllll}0 & 1 & 2 & 3 & 4\end{array}$

8. Eu não me zango facilmente.

$\begin{array}{lllll}0 & 1 & 2 & 3 & 4\end{array}$

9. Raramente eu espero que coisas boas aconteçam comigo.

$\begin{array}{lllll}0 & 1 & 2 & 3 & 4\end{array}$

10. De maneira geral, eu espero que me aconteçam mais coisas boas do que coisas ruins.

$\begin{array}{lllll}0 & 1 & 2 & 3 & 4\end{array}$ 